19. Melykhov A.N., Bernshteyn L.S., Korovyn S.Ya. Sytuatsyonnye sovetuyushchye systemy s nechetkoy lohykoy.M.: Nauka. Hl. red. fyz.-mat. lyt., 1990. - 272 s.;

20. Enta E. Teoryya nechetkykh reshenyy: Nechetkye mnozhestva y teoryya vozmozhnostey. Poslednye dostyzhenyya /Pod red. R.R. Yahera. - M.: Radyo y svyaz', 1986. Chast' IV. Praktycheskye zadachy. - S. 301-312.;

21. Pyt'ev Yu.P. Vozmozhnost'. Elementy teoryy y prymenenyya. - M.: Edytoryal URSS, 2000. - 192 s.;

22. Shleer S., Mellor S. Obъektno-oryentyrovannyy analyz: Modelyrovanye myra v sostoyanyyakh. - K.: Dyalektyka, 1993. - $240 \mathrm{~s}$;

23. Yoffe A.F. Personal'nye EVM v orhanyzatsyonnom upravlenyy. - M.: Nauka. Hl. red. fyz.mat. lyt., 1988. - 208 s.

\title{
НАУКОМЕТРИЯ В РАМКАХ ЕВРОИНТЕГРАЦИОННЫХ ПРОЦЕССОВ УКРАИНЫ
}

\author{
Егоров B.6. ${ }^{1}$ \\ ${ }^{1}$ Одесская национальная академия пищевых технологий, Одесса \\ E-mail: yegorov-victor@mail.ru \\ ORCID: $\underline{0000-0003-4699-834 \mathrm{X}}$
}

Copyright (C) 2014 by author and the journal "Automation technological and business - processes”. This work is licensed under the Creative Commons Attribution International License (CC BY). http://creativecommons.org/licenses/by/4.0/

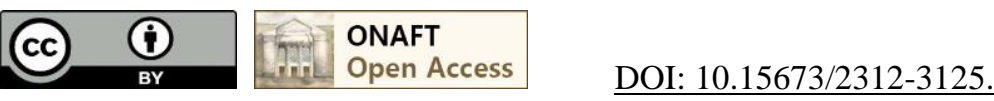

\begin{abstract}
Аннотация
Рассмотрены недостатки различных наукометрических показателей и спорность их необходимости. Рассмотрены недостатки наукометрических показателей, указано, что максимум внимания должно быть уделено непосредственно нивелированию их как таковых. Все подобные показатели можно условно разделить на 3 типа: основанные на количестве публикаций, основанные на количестве цитирований и комбинированные показатели (основаны на количестве цитирований и количестве публикаций одновременно).
\end{abstract}

\section{Abstract}

Shortcomings of various scientometric indicators and argumentativeness of their need are considered. Shortcomings of scientometric indicators are considered, is specified that a maximum of attention has to be paid directly to their leveling as those. All similar indicators can be divided into 3 types conditionally: the indicators based on number of publications, based on number of citings and combined (are based on number of citings and number of publications at the same time).

Ключевые слова

Наукометрия, наукометрические показатели, индексы цитирования, рефератиные базы данных, научные публикации

Введение. Ввиду непрерывно увеличивающегося количества ученых и сфер их научных изысканий все более актуальной проблемой становится поиск средств сравнения «успешности» ученых между собой. Сам факт вхождения ученого в Английское Королевское научное общество, например, или получение престижной, всемирно 


\section{ОГЛЯДИ ТА РЕКЛАМНІ МАТЕРІАЛИ}

известной Нобелевской премии или престижнейших Международных премий (Абелевская и Филдовская в математике или премия Тьюринга в информатике и Притцкеровская в архитектуре) объективно как меры успешности ученого стали малоэффективными из-за абсолютности и чрезвычайно малого количества тех, кто удостоен чести их получения. Для тех, кому до получения таких наград и премий еще далеко, а азарта «померяться силами» вместе с тем не меньше, существуют различные наукометрические показатели. Цель наукометрических исследований - дать объективную картину развития научного направления, оценить его актуальность, потенциальные возможности, законы формирования информационных потоков и распространения научных идей. Реализация этой цели включает в себя ряд конкретных задач, совокупное решение которых должно дать ответ на большинство поставленных вопросов. Серьезный вклад в создании методологии и инструментария наукометрии внес Ю. Гарфилд, который в 1955 году высказал идею об индексировании ссылок на представленные в пристатейной библиографии публикации. Основополагающая идея наукометрических рейтингов основана на поверхностном взгляде на процесс получения научного результата. Все подобные показатели можно условно разделить на 3 типа: основанные на количестве публикаций, основанные на количестве цитирований и комбинированные показатели (основаны на количестве цитирований и количестве публикаций одновременно)

Показатели, основанные на количестве публикаций в целом малоэффективны, прежде всего, из-за очевидной субъективности. Оценивая результативность ученых по таким показателям, молодые ученые будут всегда в заранее проигрышном положении по сравнению со своими старшими коллегами. С целью нивелирования возраста ученого предлагался и относительный показатель, рассчитываемый как отношение общего количества публикаций и научного стажа автора. По такому показателю абсолютным рекордсменом можно считать советского химика Стручкова Ю.Т., который за период с 1981 по 1990 гг. опубликовал 948 статей, т.е. в среднем 4 дня на статью. Известен также и американский профессор Э. Тополь, который в период с 1980 опубликовал 1702 статьи, т.е. в среднем 7 дней на статью. Во многом такому положению дел способствуют и «холодные» авторы, т.е. включенные в состав авторов за свой авторитет и косвенную причастность к научному коллективу. Так, известно более 80-ти случаев, когда одновременно соавторами статьи являлось более 3000 человек и одна статья Института физики высокий энергий из Протвино, авторами которой значится 3185 человек [1].

Показатели, основанные на количестве цитирований - индексы цитирования (как правило, отражают суммарное количество ссылок в научных публикациях на работы автора). Индекс цитирования в целом отражает реакцию научного сообщества на соответствующие публикации. В основе таких показателей лежит предположение, что плохие работы не цитируют, за исключением особых отношений между авторами. Цитируемость зависит не только от уровня научных результатов, но и от других факторов, например, своевременности. Длительное время очень низкой будет цитируемость публикаций с научными результатами, которые значительно опередили текущие потребности или возможности их использования [1].

Большинству индексов цитирования свойственны такие особенности:

а) игнорируют самоцитирование или цитирование соавторами, что существенно снижает рейтинг «ученого-затворника» публикации которого интересуют только его самого;

b) игнорируют повторные цитирования одной работы одним тем же ученым, что уменьшает влияние договорного цитирования;

c) учитывают личный вклад ученого, разделяя количество цитирований между соавторами;

d) учитывают репутацию цитирующего издания, взвешивая количество ссылок в журнале на его фактор авторитетности;

е) учитывают интенсивность цитирований в разных науках (в биологии в 8 раз выше, чем в математике [2]).

При этом, кроме прямых ссылок на конкретную статью в списке литературы также имеет место скрытое и неформальное цитирование - т.е. ссылка на конкретный труд непосредственно в тексте публикации, без ее дальнейшего упоминания в списке литературы. Приводятся наблюдения [3], согласно которым через 10-30 лет после публикаций статей-шедевров на них все чаще начинают ссылаться неформально.

Показатели, основанные на количестве публикаций и на количестве цитирований одновременно в основе цели своего создания содержат задачу выявления ученых, которые пишут много и качественно. Одним из таких показателей является индекс Хирша. Индекс Хирша - показатель, предложенный в 2005 году аргентиноамериканским физиком Хорхе Хиршем из Калифорнийского университета в Сан-Диего первоначально для оценки научной продуктивности физиков. Индекс Хирша является количественной характеристикой продуктивности учёного, группы учёных, научной организации или страны в целом, основанной на количестве публикаций и количестве цитирований этих публикаций. Индекс Хирша или h-индекс - это максимальное целое число h, указывающее, что автор опубликовал $\mathrm{h}$ статей, каждая из которых процитирована хотя бы $\mathrm{h}$ раз. Эти $\mathrm{h}$ статей 
составляют ядро Хирша или h-ядро. Чтобы попасть в ядро Хирша, статью должны процитировать хотя бы $\mathrm{h}$ раз. Чтобы получить высокий индекс Хирша, надо писать много, при этом, не дробя результаты по нескольким публикациям. Простота расчетов и нечувствительность к типовым приемам искусственного улучшения вышерассмотренных показателей мгновенно сделали индекс Хирша популярным наукометрическим индикатором.

Недостатки индекса Хирша связаны с тем, что в нем не учитываются: 1) насколько превышен порог цитирований в ядре Хирша; 2) длина «хвоста», т.е. количество публикаций, не вошедших в ядро и уровень их цитирования. Для компенсации этих недостатков предложены более тридцати модификаций индекса Хирша.

Далее приведены лишь некоторые из них:

- Individual $\boldsymbol{h}$-index (original) - результат деления стандартного $h$-индекса на среднее число авторов в статьях, которые входят в Хирш-ядро публикаций. Этот показатель призван уменьшить влияние на $h$ индекс числа соавторов публикаций, которое, по статистике, существенно отличается в различных областях знаний;

- Individual $\boldsymbol{h}$-index (PoP variation) - вычисление $h$-индекса когда вместо полного числа цитирований каждой статьи используется отношение числа цитирований к числу авторов публикации;

- $g$-Index - индекс, учитывающий статьи ученого с наибольшим цитированием, который определяется следующим образом: Наибольшее иелое число $g$ публикаций, которые все вместе набрали $g^{2}$ и более цитирований. (Исправляет недостаток индекса Хирша, который можно сформулировать следующим образом: «если статья попадает в число наиболее цитируемых $h$ статей, то цитирование этой конкретной статьи больше никак не учитывается»);

- $\boldsymbol{a}$-Index - это просто среднее число ссылок на статьи, входящие в Хирш-ядро;

- $\boldsymbol{m}$-Index - это медиана числа цитирований $h$ статей, входящих в Хирш-ядро публикаций автора. Является некоторым вариантом $a$-индекса и попыткой учесть распределение числа цитирований статей, входящих в Хирш-ядро;

- $\quad$ i- Index - научная организация имеет индекс i, если не менее і учёных из этой организации имеют индекс Хирша не менее і (i-индекс $=20$ означает, что не менее 20 ученых имеют индекс Хирша 20).

Однако всем рассмотренным индексам свойственны существенные недостатки [3]:

a) так как, наукометрические показатели легко вычислить, то велик риск их неадекватного использования в качестве единственного критерий оценки многогранной научно - исследовательской деятельности ученого;

b) использование наукометрических показателей в качестве критериев оценки научной деятельности провоцирует ученых к «накрутке» этих показателей различными способами.

Кроме того, следует также отметить и другой не менее важный недостаток. В основе своей все рассмотренные индексы цитирования базируются на предположении, что автор честно указывает других авторов, на положения которых ссылается в своем собственном научном труде, в противном случае он рискует быть обвинен в плагиате и согласно Закону об авторских и смежных правах быть притянутым к ответственности. Из выше приведенного вытекают как минимум две существенных проблемы:

a) Согласно упомянутому Закону крайне трудно притянуть кого-либо к реальной ответственности за плагиат. Специалисты отмечают, что, несмотря на значительное увеличение количества преступлений данной категории, эффективность борьбы с ними продолжает оставаться на невысоком уровне. Суды рассматривают лишь 2\% от общего числа возбужденных дел, а почти $98 \%$ прекращается на стадии предварительного расследования [5]. Качество применения норм уголовного закона по делам о нарушении авторских и смежных прав продолжает оставаться весьма и весьма низким [6].

b) Второй важной проблемой остается сквозное цитирование. Чтобы проще описать суть проблемы - выше намеренно приведен пример, который без дополнительного акцентирования внимания так и остался бы незамеченным. Для того, чтобы описать проблему о низкой статистике реальной ответственности за плагиат, о которой шла речь в предыдущем пункте, была использована статья [7], авторами которой, в результате самостоятельно проведенной статистической работы, были представлены удобные цифры, которые и легли в свою очередь в описание пункта а) выше. Однако указанные там же источники информации [5] и [6] были источниками в работе [7], но сама работа [7] отмечена в списке не была (изначально). Таким образом, явной становится проблема, когда одним автором проводится глобальная объемная аналитическая работа, приводятся свои источники литературы, однако, зачастую, в список литературы другими приводятся лишь источники этой работы, оставляя за полем зрения читателей автора первичного аналитического обобщающего труда, выдавая их при этом за результат собственного 


\section{ОГЛЯДИ ТА РЕКЛАМНІ МАТЕРІАЛИ}

исследования. В работе [1] даже приводится занятный пример обнаружения таких фактов, когда в списках литературы от статьи к статье «кочует» одна и та же изначально допущенная опечатка, что напрямую свидетельствует о том, что авторы не читали оригинала и приводят работу уже как источник непосредственно своих изысканий, лишь потому, что та значилась источником в работе которая, по сути воруется.

Кроме ряда очевидных недостатков различных наукометрических показателей упоминаются также мнения, что погоня за оценкой цитируемости отечественных работ является лишь стимулом для того, чтобы все работы переводили на английский язык, для удобства зарубежных ученых и удобства различных спецслужб. Также присутствуют мнения, что вся погоня за наукометрическими показателями среди ученых Мира косвенными методами намеренно провоцируется представителями основных глобальных систем Web of Science (WoS) компании Thomson Reuters (США) и Scopus компании Elsevier (Голландия) т.к. это в свою очередь стимулирует отечественные журналы вступать в эти системы, что конечно очень не просто для последних в виду высоких требований предъявляемых к журналам - претендентам, но и очень не дешево.

В виду рассмотренных недостатков наукометрических показателей авторы [4] предлагают отказаться от практики использования при оценке вклада ученого в науку различных искусственных показателей и даже предлагают ряд альтернативных мер:

1. Следует вновь разделить «перечень ВАК» на «докторский» (достаточно краткий, не более $10 \%$ от текущего списка) и «кандидатский»;

2. Следует восстановить на новой основе использовавшееся в СССР ранжирование научных издательств на «центральные» и «региональные»;

3. При проведении научных конференций следует внедрить практику подведения итогов с выделением авторов нескольких лучших докладов;

4. Дополнить пару «Доктор наук» - «Кандидат наук» третьей составляющей «Заслуженный доктор наук».

Однако очевидным в предложенных мерах является тот факт, что в процессе определения за автором права публикации в «центральном» научном издании или получения статуса лучшего доклада и прочих, также присутствует субъективный фактор личного мнения (личного отношения к автору) многоуважаемых членов организационного комитета конференции или редакционной коллегии журнала.

Выводы: Несмотря на рассмотренные недостатки различных наукометрических показателей и генерируемую спорность их необходимости, а также учитывая возможности «намеренного накручивания» этих показателей, очевидным является все же их необходимость как раз в первую очередь из-за их стремления к объективности. Если работа качественная, кто бы не был ее автор, а ее результаты представляют ценность для продвижения разработок других ученых, предполагая конечно честность последних, будут иметь место ссылки на указанную работу, а стало быть, увеличение цитируемости первоначального автора. Безусловно, рассмотренные недостатки наукометрических показателей существенны и максимум внимания должно быть уделено непосредственно нивелированию их как таковых. В конце концов, важным является не количество публикаций и даже не надуманная респектабельность изданий, в которых они публикуются, а то, что именно заложено в публикации, и от того на сколько оно имеет ценность для развития науки, что и будет в свою очередь отражено в количестве ссылок и цитирований на труд. Не будем забывать, что когда в 1905 году Альберту Эйнштейну присвоили докторскую степень, его диссертация оказалась абсолютным рекордом краткости среди ученых всех времен, когдалибо защищавших диссертации: несколько страниц рукописного текста, в основном формулы, из которых важнейшая обессмертила его имя: "энергия равняется массе, помноженной на квадрат скорости света". Это фундамент новой науки.

\section{Литература}

1. Штовба С.Д., Штовба Е.В. Обзор наукометрических показателей для оценки публикационной деятельности ученого. Управление большими системами, Специальный выпуск 44: «Наукометрия и экспертиза в управлении наукой», 262 - 278 C.;

2. PODLUBNY I. Comparison of scientific impact expressed by the number of citations in different fields of science // Scientometrics. - 2005. - Vol. 64, №.1. - P. 95-99.;

3. Писляков В.В. Наука через призму статей // Публичные лекции «Полит.ру». - 2011. - [Электронный ресурс] URL: http://polit.ru/article/2011/12/21/pislyakov_2011/ (дата обращения 27.06.2013); 
4. Орлов А.И. Наукометрия и управление научной деятельностью. Управление большими системами, Специальный выпуск 44: «Наукометрия и экспертиза в управлении наукой», 538 - 568 С.;

5. Мухина Р. Р. Методика расследования преступлений, нарушающих авторские и смежные права на аудиовизуальные произведения: Автореф. дис. канд. юрид. наук. Томск, 2010. С. 1-2;

6. Алиев В. М., Борисов А. В. О наиболее распространенных ошибках, допускаемых при квалификации преступления, связанного с нарушением авторских и смежных прав // Рос. следователь. 2011. № 2. С. 19-22;

7. Ершов О.Г., Карпов К.В. Особенности квалификации преступления о нарушении авторских и смежных прав. Журнал «Уголовный кодекс» №2, 2014;

\section{References}

1. Shtovba S.D., Shtovba E.V. Obzor naukometricheskih pokazateley dlya otsenki publikatsionnoy deyatelnosti uchenogo. Upravlenie bolshimi sistemami, Spetsialnyiy vyipusk 44: «Naukometriya i ekspertiza v upravlenii naukoy», 262 - 278 S.;

2. PODLUBNY I. Comparison of scientific impact expressed by the number of citations in different fields of science // Scientometrics. - 2005. - Vol. 64, \#.1. - P. 95-99.;

3. Pislyakov V.V. Nauka cherez prizmu statey // Publichnyie lektsii «Polit.ru». - 2011. - [Elektronnyiy resurs] URL: http://polit.ru/article/2011/12/21/pislyakov_2011/(data obrascheniya 27.06.2013);

4. Orlov A.I. Naukometriya i upravlenie nauchnoy deyatelnostyu. Upravlenie bolshimi sistemami, Spetsialnyiy vyipusk 44: «Naukometriya i ekspertiza v upravlenii naukoy», 538 - 568 S.;

5. Muhina R. R. Metodika rassledovaniya prestupleniy, narushayuschih avtorskie i smezhnyie prava na audiovizualnyie proizvedeniya: Avtoref. dis. kand. yurid. nauk. Tomsk, 2010. S. 1-2;

6. Aliev V. M., Borisov A. V. O naibolee rasprostranennyih oshibkah, dopuskaemyih pri kvalifikatsii prestupleniya, svyazannogo s narusheniem avtorskih i smezhnyih prav // Ros. sledovatel. 2011. \# 2. S. 19-22;

7. Ershov O.G., Karpov K.V. Osobennosti kvalifikatsii prestupleniya o narushenii avtorskih i smezhnyih prav. Zhurnal «Ugolovnyiy kodeks» \#2, 2014;

\section{КРАТКОЕ ОПИСАНИЕ ТИПОВОГО ТЕХНИЧЕСКОГО РЕШЕНИЯ ОПЕРАТИВНОЙ БЛОКИРОВКИ ЭЛЕКТРИЧЕСКОЙ ПОДСТАНЦИИ НА БАЗЕ ОБОРУДОВАНИЯ РНОЕNIX СОNTАСТ}

\section{ООО «ФЕНИКС КОНТАКТ»}

Copyright (C 2014 by author and the journal “Automation technological and business - processes". This work is licensed under the Creative Commons Attribution International License (CC BY). http://creativecommons.org/licenses/by/4.0/
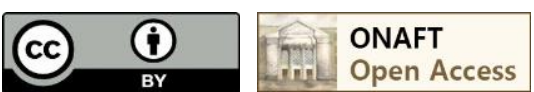

DOI: $10.15673 / 2312-3125$.

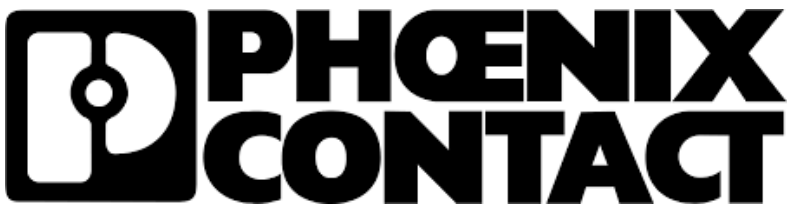

Структура. Структура решения на базе оборудования Pheonix Contact представлена в приложении 1 и построена с помощью сети PROFINET -IO. PROFINET-IO предоставляет множество преимуществ, как для системных интеграторов, так и для производителей машин и оборудования.

Сочетая в себе известную простоту использования сети PROFIBUS-DP и стандартный физический уровень сети Ethernet, сеть PROFINET-IO отличается более высокой скоростью связи, более простым управлением информацией об устройствах, а также открытостью для приложений, использующих стандартные коммуникационные сервисы Ethernet. B PROFINET-IO предусмотрены стандартизованные функции для 